Conflict of interest: Lung

Therapeutics Inc. (LTI) sponsored the trial. SI serves as a Founder and Chief Scientific Officer of LTI and a member of the Board of Directors of $\mathrm{LTI}$, which provided funding for preparation of the drug product and for the trial. He has an equity position (first-tier conflict of interest) in the company, as does the University of Texas Horizon Fund and the UTHSCT. He has a conflict-of-interest plan acknowledging and managing these declared conflicts of interest through the UTHSCT and the UT System. He is an inventor on a patent (USPTO 7332469) held by the UT Board of Regents and licensed to LTI. AAK and GF have received funding from LTI for this study, and they and KS likewise have conflict-of-interest management plans at the UTHSCT. KPS has an equity position in LTI. AAK, GF, and SI are inventors on patent USPTO 10,175,255 B2. NR and YCCL serve as key opinion leaders for LTI, and NR is paid for input to the company. SS is a paid consultant for $\mathrm{LTI}$, and JG received payment as medical monitor for the study from LTI. LB has a potential conflict of interest, having more than $\$ 50,000$ funding support for personnel and laboratory work for this study from LTI. AMS received funding in the fiscal year prior to submission greater than $\$ 50,000$ from CSK for 2 funded COPD trials. YCCL has led clinical trials for which Rocket Ltd. provided pleural drainage kits for patients without charge. YCGL has served as advisor to LTI and CareFusion/BD.

Copyright: (c) 2019 American Society for Clinical Investigation

Submitted: January 19, 2019

Accepted: April 12, 2019

Published: May 16, 2019.

Reference information: JCI Insight. 2019;4(10):e127470. https://doi. org/10.1172/jci.insight.127470.

\section{Phase I trial of the single-chain urokinase intrapleural LTI-01 in complicated parapneumonic effusions or empyema}

\author{
Lutz Beckert, ${ }^{1}$ Ben Brockway, ${ }^{2}$ Graham Simpson, ${ }^{3}$ Anne Marie Southcott, ${ }^{4}$ Y.C. Gary Lee, ${ }^{5}$
} Najib Rahman, ${ }^{6}$ Richard W. Light, ${ }^{7}$ Steven Shoemaker, ${ }^{8}$ John Gillies, ${ }^{9}$ Andrey A. Komissarov, ${ }^{10}$ Galina Florova, ${ }^{10}$ Timothy Ochran, ${ }^{11}$ William Bradley, ${ }^{12}$ Harrison Ndetan, ${ }^{13}$ Karan P. Singh, ${ }^{13}$ Krishna Sarva, ${ }^{10}$ and Steven Idell ${ }^{10}$

'University of Otago, Christchurch, New Zealand. 'University of Otago Dunedin School of Medicine, Dunedin, New Zealand. ${ }^{3}$ Cairns Hospital, Cairns, Queensland, Australia. ${ }^{4}$ Western Health, Footscray, Victoria, Australia. ${ }^{5}$ Sir Charles Gairdner Hospital, Perth, Western Australia, Australia. ${ }^{5}$ Nuffield Department of Medicine, University of Oxford, and Oxford NIHR Biomedical Research Centre, Oxford, United Kingdom. 'Department of Medicine, Vanderbilt University Medical Center, Nashville, Tennessee, USA. ${ }^{8}$ Nicosof LLC, Erie, Colorado, USA. ${ }^{9}$ Clinical Network Services (CNS), Auckland, New Zealand. ${ }^{10}$ Departments of Cellular and Molecular Biology, ${ }^{11}$ nnnovation Initiative, ${ }^{12}$ Department of Radiation Oncology, and ${ }^{13}$ Department of Epidemiology and Biostatistics, School of Community and Rural Health, The University of Texas Health Science Center at Tyler (UTHSCT), Tyler, Texas, USA

BACKGROUND. Current dosing of intrapleural fibrinolytic therapy (IPFT) in adults with complicated parapneumonic effusion (CPE)/empyema is empiric, as dose-escalation trials have not previously been conducted. We hypothesized that LTI-01 (a single-chain urokinase [scuPA]), which is relatively resistant to plasminogen activator inhibitor-1 (PAI-1), would be well tolerated.

METHODS. This was an open-label, dose-escalation trial of LTI-01 IPFT at 50,000-800,000 IU daily for up to 3 days in adults with loculated CPE/empyema and failed pleural drainage. The primary objective was to evaluate safety and tolerability, and secondary objectives included assessments of processing and bioactivity of scuPA in blood and pleural fluid (PF), and early efficacy.

RESULTS. LTI-01 was well tolerated, with no bleeding, treatment-emergent adverse events, or surgical referrals ( $n=14$ subjects). Urokinase PA (uPA) antigen increased in PFs at 3 hours after LTI-01 $(P<0.01)$ but not in plasma. PF saturated active PAI-1, generated PAI-1-resistant bioactive complexes, and increased PA and fibrinolytic activities and D-dimers. There was no systemic fibrinogenolysis or increments in plasma D-dimers. Decreased pleural opacities occurred in all but 1 subject. Both subjects receiving 800,000 IU required 2 doses to relieve pleural sepsis, with 2 other subjects similarly responding at lower doses.

CONCLUSION. LTI-01 IPFT was well tolerated at these doses, with no safety concerns. Bioactivity of LTI-01 IPFT was confirmed, limited to PFs, where its processing simulated that previously reported in preclinical studies. Preliminary efficacy signals including reduction of pleural opacity were observed.

TRIAL REGISTRATION. ANZCT ID: ACTRN12616001442493.

FUNDING. Lung Therapeutics Inc. (LTI), NIH SMARTT HHSN268201100014C (SI), UO-1 HL12184101A1 (SI). 1R01HL130402-01A1 (AAK, GF, SI), UTHSCT AC18-09 (AAK). 
to hospitals in the United States and can require surgery to relieve pleural sepsis (5). Treatment entails costs of care of more than a third of a billion dollars in the United States and United Kingdom $(5,6)$, indicative of a substantive global burden.

Intrapleural fibrinolytic therapy (IPFT) has been part of the therapeutic arsenal for CPE/empyema for more than 60 years, predicated on the concept that lysis of the intrapleural, extravascular fibrinous neomatrix can pharmacologically relieve impaired drainage of purulent collections that support persistent pleural sepsis (7). While it is generally effective in children, the safety profile and efficacy of IPFT in adult patients currently remain unclear (8-10). A number of unapproved agents have been used to augment pleural drainage in adults with CPE/empyema, with variable results $(11,12)$.

We used single-chain urokinase plasminogen activator (scuPA) as the interventional agent and tested its safety after intrapleural delivery as the primary study objective. LTI-01, a new form of scuPA produced in CHO cells, is an IPFT candidate that offers a number of conceptual advantages, including relative resistance to inhibition by plasminogen activator inhibitor-1 (PAI-1), the major plasminogen activator (PA) inhibitor within pleural fluids (PFs) (13), and durable, low-grade bioavailability in PFs over 24 hours (13). Extensive analyses including good laboratory practice (GLP) toxicology studies performed as part of NHLBI SMARTT (Science Moving Towards Translation and Therapy) contract HHSN268201100014C demonstrated that this was a well-tolerated agent, including after intrapleural administration. In extensive preclinical testing, scuPA IPFT effectively cleared fibrinous collections associated with several forms of pleural injury, including empyema (13-18).

A major impediment to interpretation of all trials concerning the efficacy and safety of IPFT to date has been that the dosing of IPFT has been empiric, with dosing of all forms of IPFT subject to arbitrary rather than evidence-based selection $(19,20)$. We are unaware of any previous phase I dose-escalation trials done to establish dosing and safety as a prelude to efficacy testing in the IPFT field. Further, we are unaware of any prior reports in which PF bioactivity or processing of any of the IPFT agents was examined. Such analyses were not included in the design of the large and influential Multicenter Intrapleural Sepsis Trials 1 and 2 (MIST1 and -2) phase II studies $(4,5)$. In the present report, we sought to address these critical gaps in two ways. First, we conducted the first in-field dose-escalation trial of any intrapleural fibrinolysin and tested intrapleural recombinant LTI-01 in patients with pre hoc CPE/empyema, pleural loculation, and failure to drain. Second, we integrated first-in-kind comprehensive analyses of the intrapleural and systemic processing of LTI-01 into the protocol design. These analyses enabled us to assess the safety and PF bioactivity of dose-escalated LTI-01 IPFT in adult CPR/empyema subjects and to better understand its contribution to treatment outcomes better.

\section{Results}

Demographics. The intention-to-treat (ITT) population comprised all subjects $(n=14)$ who enrolled in the study and supplied post-screening data. The ITT population was used for summaries of all disposition, demographic, and other baseline data. The demographics of the ITT population are shown in Table 1. All 14 subjects received antibiotics prior to and at screening. After study entry, they were continued on the regimens listed in Supplemental Table 1 (supplemental material available online with this article; https://doi. org/10.1172/jci.insight.127470DS1), which also describes PF characteristics, biomarkers, and cultures. PF cultures were positive for 4 of 14 patients at study entry; 1 was not cultured; and 9 yielded no growth. Three of the 4 PF-positive cultures yielded Streptococcal species. Blood cultures were negative in all patients. All subjects had purulent $\mathrm{PF}$, positive Gram staining or culture, or $\mathrm{pH} \leq 7.2$, and had loculated $\mathrm{PF}$ by chest imaging with failure to drain over 3 hours of initial observation (Figure 1).

Bleeding events. No acute intrapleural or systemic bleeding events were observed in any of the LTI-01treated subjects. There were likewise no consistent effects on heart rate, blood pressure, respiratory rate, or temperature at all dose levels. Fever $\left(>37.4^{\circ} \mathrm{C}\right)$ was present in only 1 patient.

Treatment-emergent adverse events. Assessments were done according to the study schedule (Supplemental Table 2, study protocol). A total of 22 treatment-emergent adverse events (TEAEs) were recorded in 7 $(50.0 \%)$ of the14 subjects (Table 2$)$. No TEAE was considered to be related to study treatment, based on treating physician assessment. TEAEs did not increase with dose level, as 10 were recorded in the low-dose group (50,000 IU), compared with no such events in the high-dose group (800,000 IU). The most frequently occurring TEAEs were hypotension and postprocedural persistent drain fluid (3 events each), and pleuritic pain ( 2 subjects each). Three TEAEs occurring during active study participation were classified as severe, 
including metastatic squamous cell carcinoma, sinus tachycardia, and lung abscess, and 11 were classified as moderate. There were 2 further TEAEs of note, neither attributed to LTI-01. One subject (200,000 IU) had a postprocedural hematoma before dosing on day 3 . That was considered unlikely to be related to the study drug by the local investigator and thought to be related to drain insertion. The other (50,000 IU) had sinus tachycardia. There were no life-threatening TEAEs, and none led to study discontinuation.

Serious adverse events. Serious adverse events (SAEs) were collected from the time of first study dose to day 28, hospital discharge, or death, whichever came first (Supplemental Table 3). Two subjects experienced SAEs, both leading to death, with neither death attributed to administration of LTI-01. One subject (50,000 IU) with clinical empyema and failure to drain at enrollment died from metastatic squamous cell carcinoma on day 20. This subject had the condition in addition to CPE, given that malignant cells were found in his PF after entry. A second subject (400,000 IU) has a ruptured lung abscess and died of a contralateral pneumonia on day 86 . This subject had a cavitating lung lesion of the right lower lobe, pneumonia, empyema, and obstruction of the bronchus intermedius, as well as bile duct obstruction at entry. No additional SAEs occurred after day 28 or after discharge as of database lock (May 10, 2018). No protocol deviations were considered to affect study objectives or the integrity of the data (see Section 1 in the supplemental material)

Treatment compliance. Six of the 14 subjects did not receive dosing on day 3. In accordance with the protocol, second or third LTI-01 doses could be withheld if complete resolution of the pleural process occurred after the prior dose. In one patient in the 50,000 IU group, treatment was stopped after 2 doses of LTI-01, after which tissue PA (tPA) or tPA/DNase was administered on days 3-5 by the treatment team. In a subject in the 100,000 IU group, LTI-01 was not given on day 3 because of an elevated prothrombin time (PT) of 16 seconds. This subject received tPA/DNase on days 5-8 because of an increased leukocyte count and increased $\mathrm{C}$-reactive protein (CRP). Another patient in this group did not receive the third dose of LTI-01 because of chest tube migration and required no further therapy. The subject in the 200,000 IU group with a chest wall hematoma did not receive the third dose because of clinical improvement following LTI-01 IPFT. In a patient in the 800,000 IU group, it was felt that a third dose was likewise not warranted because of radiographic/clinical improvement. In the other subject in this group, a partial third dose was given because of chest tube blockage, and the patient required no further IPFT.

Clinical laboratory testing. Mean hemoglobin decreased at all time points for the pooled subject population, while total leukocyte and neutrophil counts generally decreased during and after LTI-01 IPFT (Supplemental Figures 1-3). There was no consistent effect or effect over time of LTI-01 IPFT on mean PT, mean activated partial thromboplastin time (aPTT), or mean international normalized ratio (INR) values. Plasma fibrinogen never fell below the top normal laboratory range levels in any subject, indicating no evidence of systemic fibrinogenolysis (Supplemental Figure 4). There were no changes in blood chemistry attributable to LTI-01 IPFT (data not shown).

Preliminary assessments of efficacy. No subject was referred to surgery. None experienced a life-threatening event prior to discharge. All but one subject with underlying pleural malignancy identified after entry had a relative decrease in pleural density area or volume during the study (Table 3). Total PF drainage volumes were measured daily and exhibited increased volumes that tended to increase with dose. Total PF drainage volumes from day 1 to 23 hours after the final dose or on day 4, whichever came first, were 248.7, 525.3, 651.7, 1299.8, and 1585.0 $\mathrm{ml}$ for the LTI-01 50,000, 100,000, 200,000, 400,000, and 800,000 IU dose levels, respectively. Mean plasma CRP decreased at all time points for the pooled subject population. A maximum decrease from baseline concentrations was found by days 7-28 at $-125.4 \mathrm{mg} / 1$, with a rate of decrease trending lower during the treatment phase (Supplemental Figure 5).

Follow-up at 3 and 12 months after LTI-01 IPFT. Data were available at 3 months for all 12 patients known to survive beyond 86 days. At 3 months, no additional deaths occurred, nor were there any further hospitalizations for pleural disease, including surgery. Information was available for 10 of 12 subjects at 12 months, with no referrals to surgery or hospitalizations.

Pharmacokinetic and pharmacodynamic analyses, and intrapleural processing of LTI-01. uPA-related antigen (labeled as urokinase) data are provided in Supplemental Table 4. PF uPA antigen levels (Figure 2A; pharmacodynamic $[\mathrm{PD}])$ at 3 hours after delivery were markedly elevated when compared with preinjection levels $(P<0.05)$. They ranged from a mean day 1 postdose ( 3 hour) level of $376,830 \mathrm{pg} / \mathrm{ml}$ in the 50,000 IU dose group to $2,129,776 \mathrm{pg} / \mathrm{ml}$ in the $800,000 \mathrm{IU}$ dose group (Figure $2 \mathrm{~A}$ ). The level of the uPA antigen at 23 hours (day 2 predose) was also higher $(P<0.05)$ than that before treatment (day 1 predose). There was no 
Table 1. Baseline demographics for all subjects (ITT population)

\begin{tabular}{|c|c|c|c|c|c|c|c|}
\hline Parameter & Statistics & $\begin{array}{l}\text { LTI-01, 50,000 IU } \\
\quad(n=3)\end{array}$ & $\begin{array}{l}\text { LTI-01, 100,000 IU } \\
(n=3)\end{array}$ & $\begin{array}{c}\text { LTI-01, 200,000 IU } \\
\qquad(n=3)\end{array}$ & $\begin{array}{l}\text { L LTI-01, } 400,000 \text { IU } \\
\qquad(n=3)\end{array}$ & $\begin{array}{l}\text { LTI-01, 800,000 IU } \\
(n=2)\end{array}$ & $\begin{array}{l}\text { All LTI-01 } \\
(n=14)\end{array}$ \\
\hline \multicolumn{8}{|c|}{ ( } \\
\hline Female & $n(\%)$ & $1(33.3 \%)$ & - & - & $1(33.3 \%)$ & $1(50.0 \%)$ & $3(21.4 \%)$ \\
\hline Male & $n(\%)$ & $2(66.7 \%)$ & $3(100.0 \%)$ & $3(100.0 \%)$ & $2(66.7 \%)$ & $1(50.0 \%)$ & $11(78.6 \%)$ \\
\hline Age (yr) & Mean & 59.3 & 52.3 & 69.0 & 75.7 & 70.0 & 64.9 \\
\hline Age (yr) & Min & 52 & 42 & 57 & 72 & 51 & 42 \\
\hline BMI (kg/m²) & Min & 22.4 & 17.4 & 15.9 & 25.1 & 24.0 & 15.9 \\
\hline BMI $\left(\mathrm{kg} / \mathrm{m}^{2}\right)$ & Max & 27.5 & 33.1 & 24.7 & 27.9 & 24.3 & 33.1 \\
\hline \multicolumn{8}{|l|}{ Race } \\
\hline White & $n(\%)$ & $3(100.0 \%)$ & 2 (66.7\%) & $2(66.7 \%)$ & $3(100.0 \%)$ & $1(50.0 \%)$ & $11(78.6 \%)$ \\
\hline Other ${ }^{A}$ & $n(\%)$ & - & $1(33.3 \%)$ & $1(33.3 \%)$ & - & $1(50.0 \%)$ & 3 (21.4\%) \\
\hline
\end{tabular}

statistically significant difference $(P>0.05)$ between PF uPA antigen levels on day 1,2 , or 3 at 3 hours after treatment. These results reflect successful delivery of the LTI-01 to the pleural space, maximal detectable levels at 3 hours, and retention of the elevated uPA antigen up to 23 hours. Plasminogen-activating (PA) and fibrinolytic activities were next determined in all PF samples. As expected, changes in PF PA activity with time (Figure 2B) paralleled those observed for uPA antigen (Figure 2A). While PA activity was relatively high at 3 hours after treatment, it was at or below the limit of detection at baseline and at 23 hours after IPFT (Figure 2B). Notably, PF fibrinolytic activity was also relatively high at 3 hours after IPFT and suppressed at baseline and 23 hours (Figure 2C). PF levels of fibrin degradation products (D-dimers) (Figure 2D) increased at 3 hours after treatment and temporally correlated with changes in fibrinolytic activity. These results confirm the bioavailability of LTI-01 in PFs of treated patients with conversion into a mature 2-chain enzyme, activation of endogenous plasminogen (Supplemental Figure 6), and generation of durable fibrinolytic activity, with an increase in PF D-dimers - the products of fibrinolysis in PFs. Plasminogen levels were unchanged in plasma.

Effects of LTI-01 on changes of the fibrinolytic system in plasma. In contrast to PF (Figure 2A), plasma uPA-related antigen levels (pharmacokinetic [PK]) did not significantly increase on day 1 at 3 hours after dosing compared with before dosing (Figure $2 \mathrm{E}$ ) or at other dosing intervals. The largest increase was seen in one of the subjects dosed at 800,000 IU, with an increase of approximately $700 \mathrm{pg} / \mathrm{ml}$ after dosing (1446 $\mathrm{pg} / \mathrm{ml}$ ) compared with the predose level $(743 \mathrm{pg} / \mathrm{ml})$. Notably, uPA antigen levels in plasma (Figure 2E) were almost 3 orders of magnitude lower than those in PF at 3 hours after treatment (Figure 2A). Moreover, while the level of uPA antigen in the PF changed with time, uPA in the circulation remained stable (Figure 2E). Similar to uPA, the levels of D-dimers in plasma (Figure 2F) were up to 3 orders of magnitude lower than those in PF (Figure 2D). Plasma D-dimers did not change $(P>0.05)$ during the 3-day treatment period (Figure $2 \mathrm{~F}$ ), indicating no significant systemic changes in fibrinolytic activity.

Endogenous inhibitors affect LTI-01 and intrapleural fibrinolysis. Intrapleural concentrations of active PAI-1 in all pretreatment samples were elevated (160-200 ng/ml; Figure 3A), resulting in the observed inhibition of plasminogen activation (Figure $2 \mathrm{~B}$ ) and fibrinolysis (Figure 2C). On the other hand, the level of active PAI-1 in the majority of PF samples collected at 3 hours after treatment with LTI-01 was at or below the limit of detection (Figure 3A; $P<0.01$ versus baseline levels). Thus, PAI-1 activity at 3 hours was completely inhibited by PF LTI-01, which promotes its conversion to 2-chain uPA to activate endogenous plasminogen and thus support intrapleural fibrinolysis. Interestingly, 4 samples with elevated PAI-1 activity detected at 3 hours (one on days 1 and 3 and two on day 2; Figure 3A; post) possessed no PA activity. Thus, an increase in active PAI-1 at 3 hours likely indicates either incomplete delivery of IPFT or fast in vivo inactivation of LTI-01 in PFs of these particular patients. PAI-1 total antigen was also significantly increased in PFs (Figure 3B) versus levels found in the plasma (data not shown), reflecting overexpression of local PAI-1 in infectious pleural injury. The increase in PF levels 


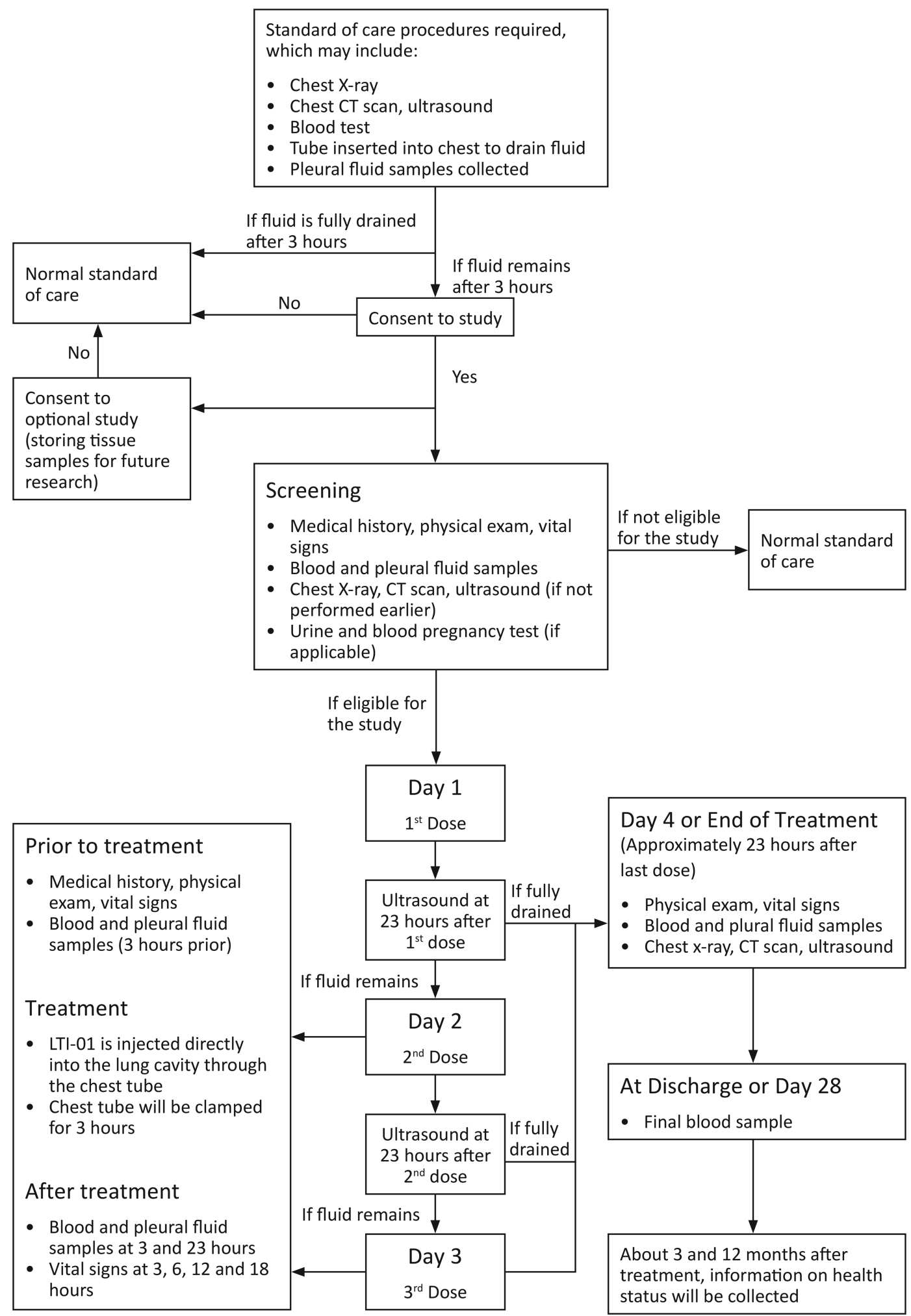

Figure 1. Protocol flow chart.

of uPA antigen at 23 hours, when compared with baseline (Figure 2A), without a significant increase in PA activity (Figure $2 \mathrm{~B}$ ) is consistent with formation of bioactive complexes with $\alpha 2$-macroglobulin (A2M), as observed in preclinical models of pleural injury (13). Indeed, levels of intrapleural A2M/ uPA complexes (Figure 3C) increased at 3 hours after IPFT and decreased at 23 hours after treatment. 
Table 2. Treatment-emergent adverse events by treatment group

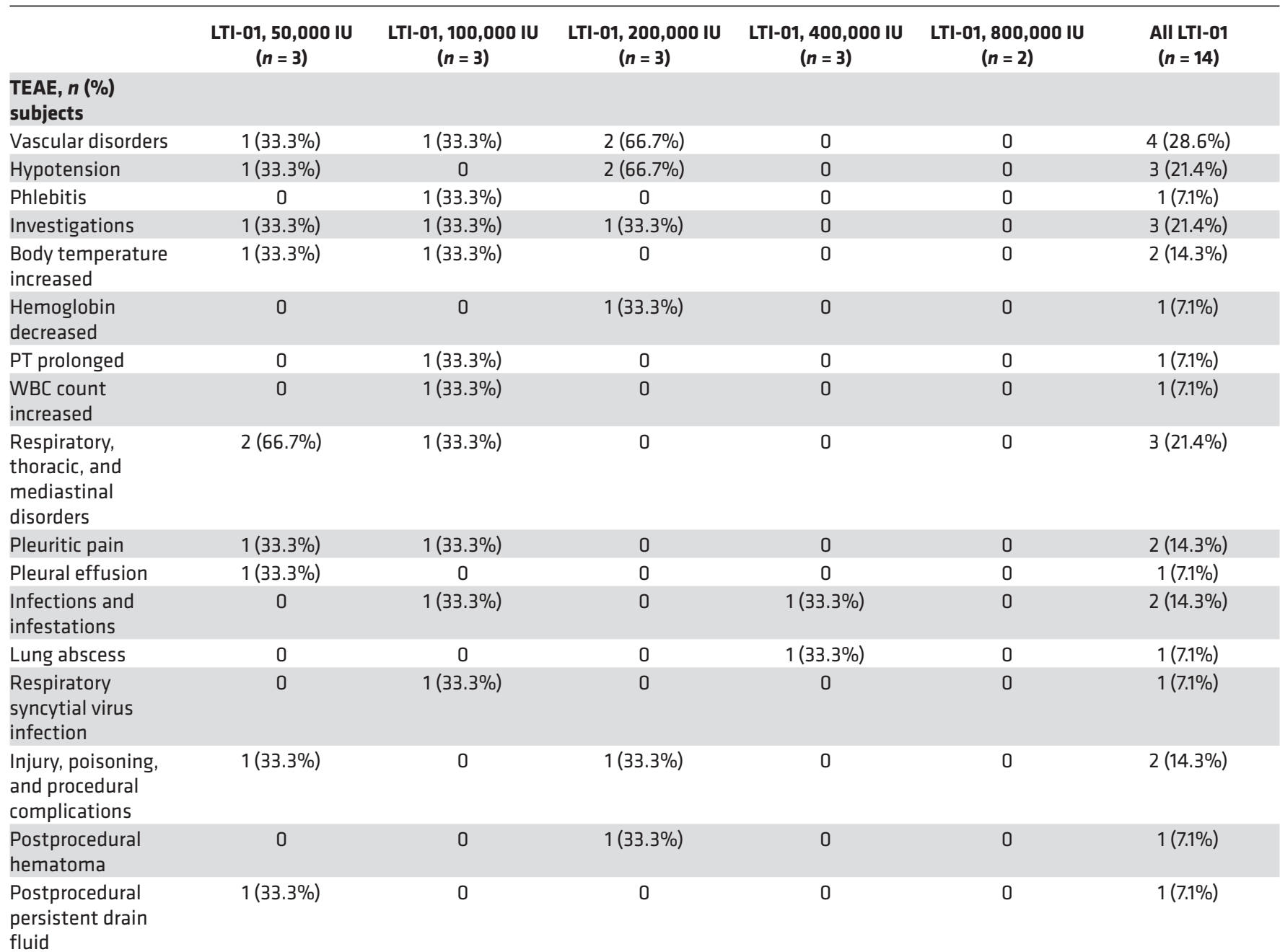

Table restricted to system organ classes (SOCs)for which $>1$ subject reported a TEAE. Numbers of events and percentages (in brackets) for each category of TEAE are presented.

Next, we tested the concept of fibrinolytic potential (FP) as a personalized predictor of the response to LTI-01 IPFT $(21,22)$. Supplementation of the predose (baseline and at 23 hours) PFs with 2-chain uPA (the FP) generated robust levels of fibrinolytic activity (median 0.7-1.3 AU; $P<0.01$ versus unsupplemented baseline levels). We evaluated PF samples collected prior to treatment and correlated the FP to changes in the pleural density area, as assessed by chest X-ray. There was a significant correlation between the FP (Figure 3D; $n=8 ; r=-0.79, P<0.05$ ) and the percent relative change of chest $\mathrm{X}$-ray pleural density area after treatment (Table 3 and Figure 4).

Individualized assessments of derangements of PF components of the fibrinolytic system and outcomes of LTI-01 $I P F T$. First, we analyzed the findings in subjects with clinical improvement proximate to LTI-01 administration without any switch to another form of IPFT or referral to surgery and reduced pleural opacification by either chest X-ray or chest CT imaging. One patient in the 400,000 IU group, 102-1005, received all 3 doses and temporally responded with clinical resolution of pleural sepsis and a relative reduction in pleural density of $45 \%$. Interestingly, this patient demonstrated the highest levels of uPA antigen, $>100 \mu \mathrm{g} / \mathrm{ml}$, in posttreatment PFs, as well as the lowest PAI-1 PF concentrations. In another patient in the 800,000IU group, 102-1007, clinical resolution of pleural sepsis was achieved with a relative reduction in pleural density of $36 \%$, so that a third dose of LTI-01 was not administered. This patient had elevated levels of PF uPA $>100 \mu \mathrm{g} / \mathrm{ml}$, with low levels of PF PAI-1 compared with other subjects (Supplemental Figure 7, A and B). In the other patient receiving 800,000 IU, only a portion of the third dose was delivered, attributable to chest tube obstruction, and 
Table 3. Pleural density area and volume changes after LTI-01 treatment

\begin{tabular}{|c|c|c|c|}
\hline Subject ID & Dose of LTI-01 (IU) & $\begin{array}{l}\text { Relative change in \% pleural } \\
\text { density area (\%) by CXR }\end{array}$ & $\begin{array}{c}\text { Relative change in pleural density } \\
\text { volume }(\%) \text { by } \mathrm{CT}^{\mathrm{A}}\end{array}$ \\
\hline $102-1001$ & 50,000 & $-85 \%$ & $-69 \%$ \\
\hline 202-1001 & 50,000 & $4 \%$ & $83 \%$ \\
\hline $202-1002$ & 50,000 & $-42 \%$ & NA \\
\hline $102-1002$ & 100,000 & $-15 \%$ & $-36 \%$ \\
\hline 103-1001 & 100,000 & $-83 \%$ & $N A^{B}$ \\
\hline 202-1003 & 100,000 & $-55 \%$ & NA \\
\hline 102-1003 & 200,000 & $-71 \%$ & $-72 \%$ \\
\hline $102-1004$ & 200,000 & $-37 \%$ & $-46 \%$ \\
\hline 202-1004 & 200,000 & NA & $-62 \%$ \\
\hline $102-1005$ & 400,000 & NA & $-45 \%$ \\
\hline 202-1005 & 400,000 & $-38 \%$ & $-38 \%$ \\
\hline $102-1006$ & 400,000 & NA & $-31 \%$ \\
\hline $102-1007$ & 800,000 & $-36 \%$ & $-58 \%$ \\
\hline $103-1002$ & 800,000 & $-85 \%$ & NA \\
\hline
\end{tabular}

${ }^{A}$ Relative change in pleural opacification: change in pleural opacification recorded as the basal opacity - posttreatment opacification divided by basal opacification, as described in Methods. ${ }^{B} \mathrm{NA}$, not available. CXR, chest X-ray.

pleural sepsis likewise resolved without further IPFT or a referral to surgery, and PF PAI-1 levels remained low at days 1 and 2 after dosing. One additional patient, 102-1001, treated with 50,000 IU/ml drained effectively, with a relative reduction in pleural density of $85 \%$ by chest X-ray, and had relatively very low levels of $\mathrm{PF}$ PAI-1 with concurrent increments of UPA and resolution of clinical pleural sepsis upon completion of LTI-01 dosing (Supplemental Figure 7, A and B). Other patients with poor outcomes remote to the treatment period had relatively elevated levels of PAI-1 or did not complete therapy, while those who responded clinically and radiographically typically had evidence of appropriate drug delivery with elevated PF uPA and reduced PAI-1 activity, as described in detail for all other subjects $(n=10)$ in Section 2 in the supplemental material and Supplemental Figure 7.

\section{Discussion}

Since the inception of $\operatorname{IPFT}(7,23)$, a number of fibrinolysins, which activate plasminogen to form plasmin and lyse fibrin, have been used to treat adults with CPE/empyema $(19,24)$. In previous meta-analyses, it was concluded that there was equivocal evidence supporting the use of IPFT for these patients $(8,9)$, with a more recent study unable to assess the impact of IPFT (10). Among factors contributing to the uncertainty, IPFT dosing remains empiric, and bioactivity of currently used IPFT agents was not confirmed in prior clinical trial testing in adults with empyema/CPE $(19,20,25)$.

In addition, to our knowledge, no form of IPFT has been approved by any regulatory agency in the United States, United Kingdom, or elsewhere to date. A major initial requirement for such approval is the performance of phase I testing in humans to establish safety. We manufactured a highly purified recombinant active pharmaceutical form of scuPA to meet requirements for regulatory approval. Initial dosing for this trial was established with GLP toxicologic studies. The drug product, LTI-01, was used in what we believe to be the first dose-escalation safety trial with CPE/empyema.

The premise for testing LTI-01 is based on its favorable biochemical properties and unique processing in PFs. Over the past 20 years, our group published several preclinical studies suggesting that scuPA was effective and well tolerated in organizing pleural injury, as reviewed recently (20). First, scuPA was found to be well tolerated in tetracycline-induced pleural injury and empyema in rabbits and cleared pleural collections without causing intrapleural or systemic bleeding (13-16). Second, scuPA IPFT is relatively PAI-1 resistant in PFs (13). This issue is of particular importance, given the characteristically high levels of PAI-1 that occur within CPE/empyema PFs $(15,26,27)$ and as confirmed in this study. While other inhibitors, including antiplasmins, are present in $\mathrm{CPE} /$ empyema $\mathrm{PFs}$, increased pleural organization associated with PAI-1 overexpression (17) attests to its impact on outcomes. We found that levels of total and active PF PAI-1 are variably increased in patients with CPE/empyema, which implies that the current empirically 
A
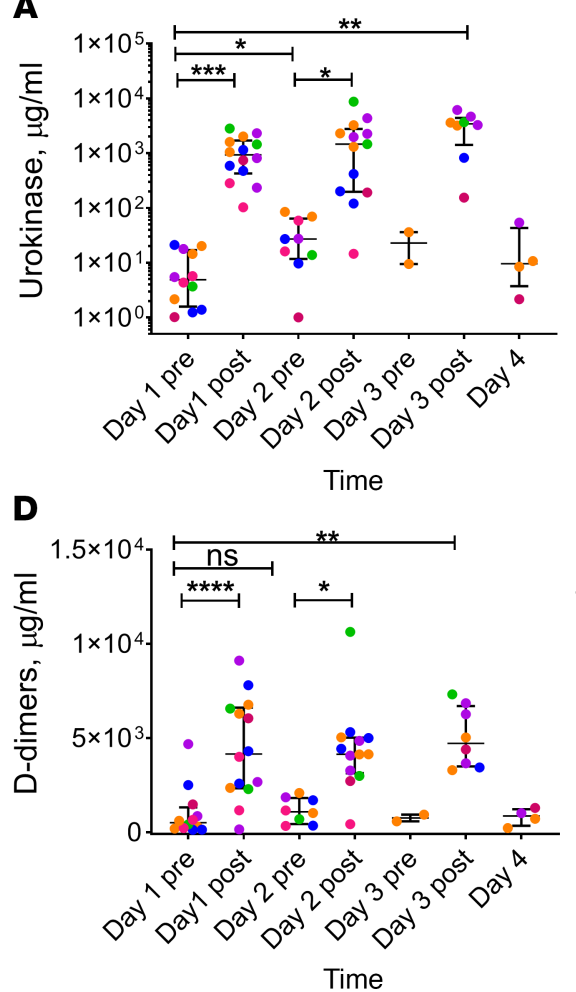

B

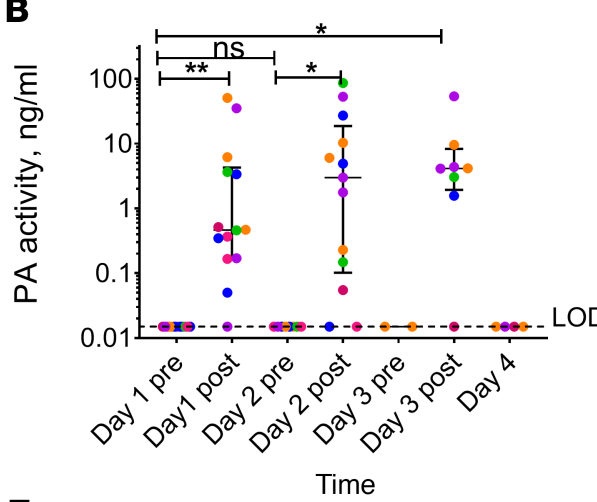

C

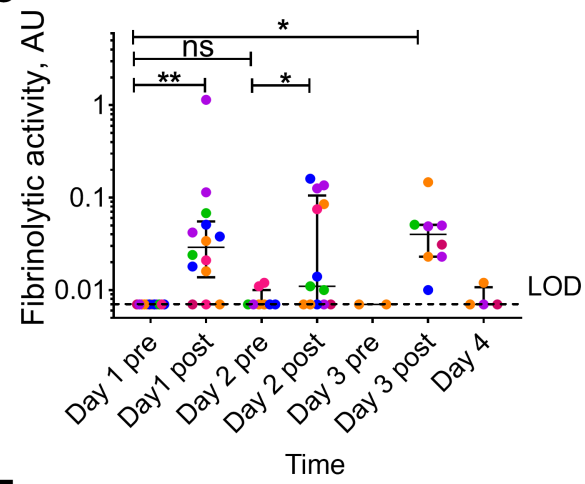

$\mathbf{E}$

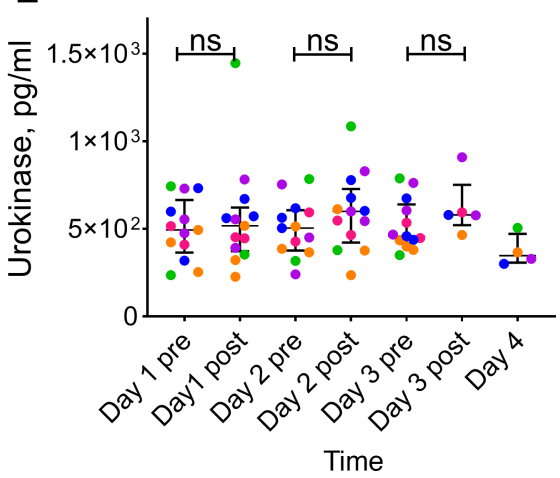

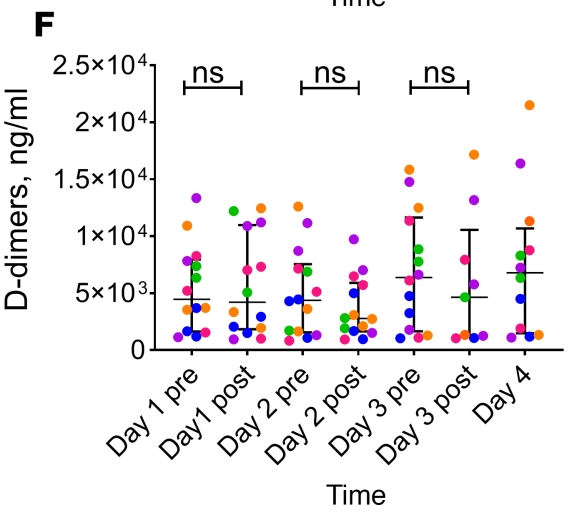

Figure 2. Biochemical responses to LTI-01 IPFT in pleural fluid and plasma. Successful delivery of LTI-01 to the pleural space (A) coincided with an increase in plasminogen activating (PA) (B) and fibrinolytic (C) activities and level of fibrin degradation product (D-dimers) (D) in pleural fluid at 3 hours after treatment, and did not affect levels of uPA antigen (E) or D-dimers in plasma (F). LTI-01 was delivered daily at doses of 50,000 (pink symbols; $n=3$ ), 100,000 (blue symbols; $n=3$ ), 200,000 (orange symbols; $n=3$ ), 400,000 (purple symbols; $n=3$ ), and 800,000 (green symbols; $n=$ 2) IU per injection. Samples of pleural fluid (day 1 before treatment [pre] $n=12$, day 1 after treatment [post] $n=14$, day 2 pre-treatment $n=9$, day 2 post-treatment $n=13$, day 3 pre-treatment $n=2$, day 3 post-treatment $n=8$, and day $4 n=4$ ) and plasma (day 1 and pre-day 3 treatment $n=14$ each, day 3 post-treatment $n=5$, and day $4 n=5$ ) were collected daily (1-4 days) just prior to each LTI-01 injection (pre) and 3 hours after (post) treatment as described in Methods. Levels of urokinase antigen were determined in pleural fluid (A) and plasma (E) using ELISA analyses (R\&D Systems). Plasminogen activating (PA) activity in pleural fluid (B) was determined by measuring the rates of activation of exogenously added human Glu-pasminogen $(200 \mathrm{nM})$ and fluorogenic plasmin substrate $(200 \mu \mathrm{M}) \mathrm{SN}-5(\mathrm{HTI})$ as previously described (21). The fibrinolytic activity in pleural fluid (C) was measured by FITC-fibrin assay, as described elsewhere (21). D-dimers were determined in pleural fluid (D) and plasma (F) using ELISA (Abcam). The plasma samples collected from the patient diagnosed with metastatic squamous cell carcinoma were excluded from the analysis. Each point represents the average of at least 2 independent measurements. Data are presented as dot plots, with horizontal lines indicating the medians and whiskers representing interquartile range. ${ }^{*} P<0.05$, ${ }^{* *} P<0.001,{ }^{* *} P<0.0001$, NS: $P \geq 0.05$, Wilcoxon's matched-pairs signed-rank test (assessed at the $5 \%$ level of significance). Dashed line indicates the lowest limit of detection (LOD) of the method.

selected IPFT dose could predispose to bleeding in some patients with relatively low PF PAI-1 or be insufficient in those with high PAI-1. This variability also may explain why LTI-01 IPFT was effective over a range of doses in individual subjects with different levels of PF PAI-1.

The demographic profile of the subjects reflects the variety of causes of pleural infection that may lead to loculation and failed pleural drainage. Subjects had a mean age of about 65 years, comparable to the cohorts previously enrolled in the MIST1 and -2 trials $(4,5)$. A limitation of the study is that only 14 of the 21 subjects that could have been enrolled actually participated, and there was no expansion of any of the cohorts as allowed by the protocol design (Supplemental Table 2, study protocol).

Two consecutive patients who received the highest dose of 800,000 IU tolerated the drug well and required only 2 doses to relieve the pleural collection. Two subjects died during the follow-up period after active study participation. Neither death was attributed to LTI-01 by the local investigator. The maximal tolerated dose (MTD) of LTI-01 IPFT was not detected. However, the absence of safety signals offers a strong rationale for further dose escalations to identify the optimal dose to be tested in the next phase of development. Higher dose escalations were not included in the study design because of limited drug supply.

There were no bleeding events, either local or systemic, in the study participants. This observation is of particular importance given reported bleeding rates of 5\%-28\% associated with different forms of IPFT $(28,29)$, 
A

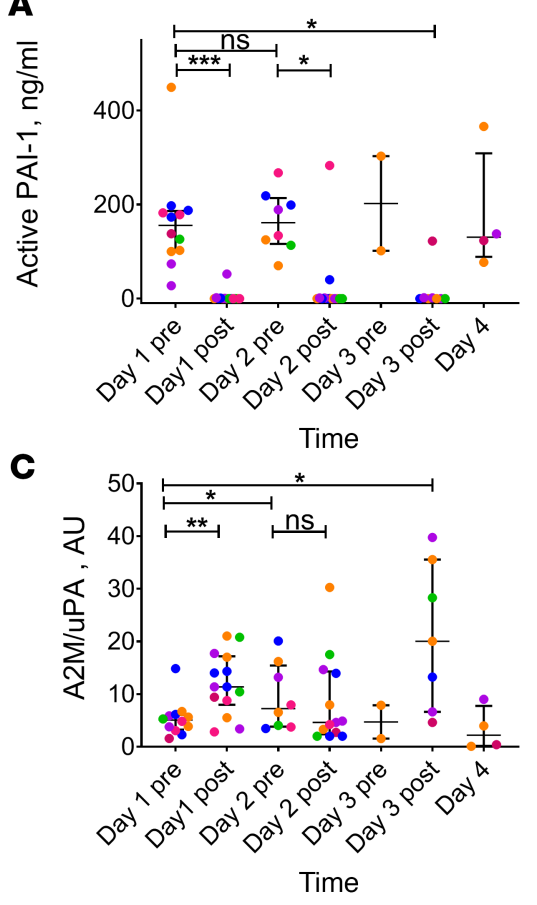

B

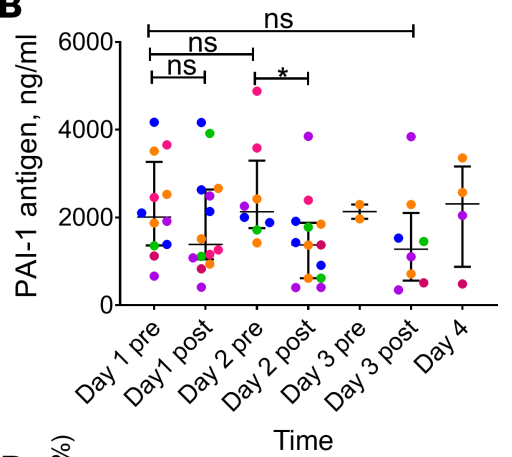

D

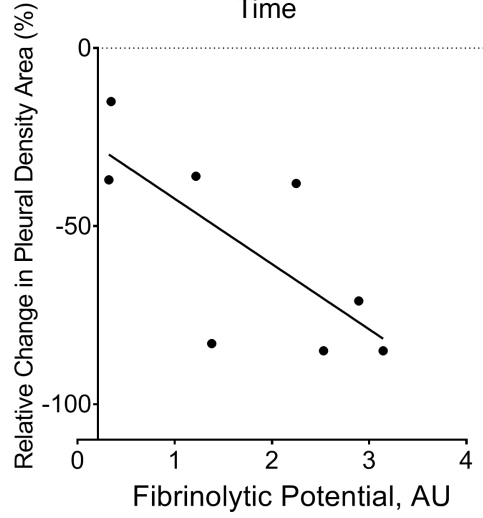

Figure 3. Inhibition of plasminogen activating and fibrinolytic activities prior to and during IPFT with LTI-01, and correlation of fibrinolytic potential with reduction of pleural opacification by chest $\mathbf{X}$-ray. Levels of plasminogen activator inhibitor 1 (PAI-1) activity (A), antigen (B), and A2M-UPA complexes (C) were determined. LTI-01 was delivered daily with doses of 50,000 (pink symbols; $n=3 ; 12$ samples), 100,000 (blue symbols; $n=3 ; 9$ samples), 200,000 (orange symbols; $n=3 ; 4$ samples), 400,000 (purple symbols; $n=3 ; 10$ samples), and 800,000 (green symbols; $n=2 ; 6$ samples) IU per injection. Samples of pleural fluid (day 1 before treatment [pre] $n=12$, day 1 after treatment [post] $n=14$, day 2 pre-treatment $n=9$, day 2 post-treatment $n=13$, day 3 pre-treatment $n=2$, day 3 post-treatment $n=8$, and day $4 n=$ 4) were collected daily (1-4 days) prior to each LTI-01 injection (pre) and 3 hours after (post) treatment as described in Methods. Levels of PAI-1 antigen and PAI-1 activity were determined in pleural fluid (A) using ELISA (Molecular Innovations). Levels of bioactive $\alpha 2$-macroglobulin-uPA (A2M-uPA) (C) complexes were determined as described elsewhere (13). Each point represents the average of at least 2 independent measurements. Data are presented as dot plots, with horizontal lines indicating the medians and whiskers representing interquartile range. ${ }^{*} P<0.05$, ${ }^{*} P<0.001$, ${ }^{* *} P$ $<0.0001$, NS: $P \geq 0.05$ using Wilcoxon's matched-pairs signed-rank test (assessed at the $5 \%$ level of significance). Correlation (D) between fibrinolytic potential (FP) and clinical outcome after IPFT with LTI-01 ( $n=8 ; r=-0.79 ; P<0.05$ by Spearman's rank correlation). FP was determined in samples of PF collected prior to IPFT as previously described $(21,22)$. Percent changes in relative pleural density on chest X-ray prior to and after IPFT with LTI-01 were calculated as previously described (5). The solid line represents the best fit of a linear equation to the data; with the percent relative changes in X-ray pleural density area (Table 3).

despite some reports of lower incidences $(12,30)$. The absence of clinical bleeding was in keeping with our preclinical experience $(11,13,14,16,18,21,31,32)$ and pretrial toxicology. The initial dose of 50,000 IU was thought to likely be subtherapeutic based on the literature $(11,33)$. However, it was intentionally used to determine how well the study drug/vehicle/known impurities (Supplemental Table 2, study protocol) were tolerated before escalating the dose. The exclusion criteria we used also had rigorous provisions designed to decrease bleeding risk associated with this trial (Supplemental Table 2, study protocol). These included abnormalities of the screening coagulation profile, known platelet dysfunction, or use of antiplatelet agents. Patients could receive deep vein thrombosis (DVT) prophylaxis but not full anticoagulation, given reports of increased bleeding risk $(34,35)$. Patients with a glomerular filtration rate of less than 30 were also excluded. Plasma fibrinogen levels were screened, as levels below $100-150 \mathrm{mg} / \mathrm{dl}$ have been linked to bleeding risk in trauma patients (36-38).

All patients maintained supraphysiologic levels of plasma fibrinogen, which is an acute-phase reactant and plasma levels remained elevated throughout the treatment period. The data indicate that administration of LTI-01 IPFT was not associated with fibrinogenolysis and suggest that future screening for fibrinogen levels does not need to be routine in the adult $\mathrm{CPE} /$ empyema population. There was no consistent effect of LTI-01 IPFT on the coagulation profiles of treated subjects. 


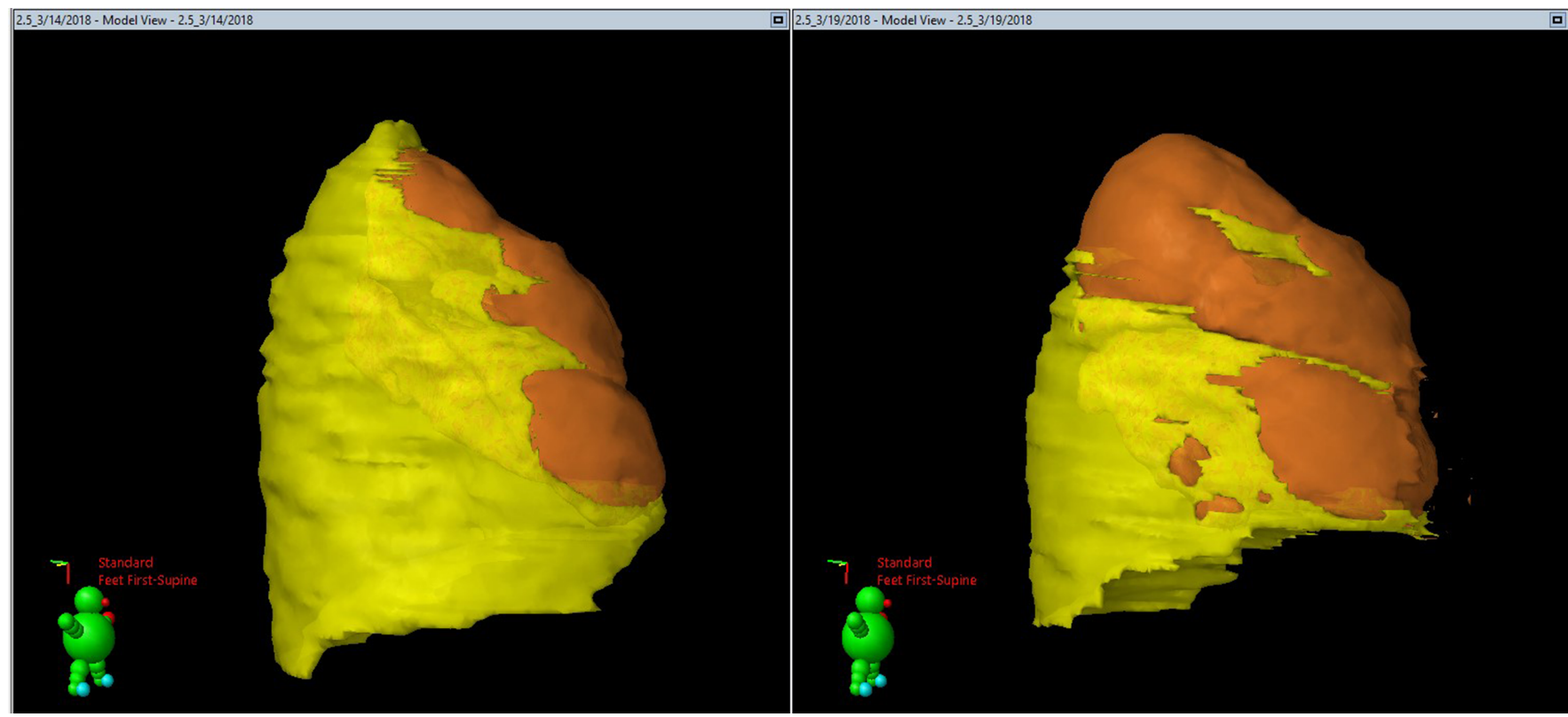

Figure 4. CT rendition from case 102-1007 (800,000 IU dosing) illustrating color mapping of the extent of pleural opacification. Pleural opacification is indicated in yellow in the lateral projection. The underlying lung is indicated in orange. The pretreatment (day -1) CT projection is illustrated in the left panel, and the posttreatment CT on day 4 is shown on the right. Calculation of the relative decrease in pleural opacification in the volume of the pleural opacification after treatment, $-58 \%$ here, was done as previously described (5). The green icon shows the orientation of each of the images, with the arrow indicating the facial orientation. The red text indicates that the patient was imaged in the supine position.

Reduced pleural opacification was generally observed by chest X-ray or chest CT imaging, using the methodology originally reported in the MIST2 trial (5). As a safety trial, this study was not powered to delineate efficacy. Therefore, this readout needs to be considered as encouraging but preliminary. PF drainage increased after LTI-01 IPFT but occurs with other forms of IPFT such as tPA/DNase IPFT (5). All baseline assessments approximated the screening or day 1 predose period, and posttreatment assessments approximated the day 4 posttreatment interval, but some were obtained at later intervals, representing another confounder. Nonetheless, LTI-01 IPFT was temporally associated with improved pleural opacification and concurrently improved the clinical status in 4 patients, including both subjects treated with 800,000 IU. These findings support advancement to additional dose escalations/group expansion. Decreases in plasma CRP levels and in white cell and neutrophil counts that generally occurred in the subjects are consistent with improvement of the underlying pleural infections. Last, the 4 subjects who received follow-up IPFT with tPA with or without DNase were all treated at one site and at the discretion of the treating team, which was permitted under the protocol.

To our knowledge, this is also the first study to assess the bioavailability and processing of IPFT in humans. PF analyses of uPA antigen (Figure 2A), PA and fibrinolytic activities (Figure 2, B and C), and D-dimers (Figure 2D) indicate that successful delivery of LTI-01 resulted in activation of endogenous plasminogen and retention of elevated intrapleural PA and fibrinolytic activities up to 3 hours after the treatment. No systemic activation of fibrinolysis was detected (Figure 2, E and F). Notably, both PF PA and fibrinolytic activities were inhibited by 23 hours after LTI-01 IPFT, supporting the use of daily dosing. Inhibition of fibrinolysis at 23 hours was accompanied by an increase in the level of intrapleural active PAI-1 (Figure 3A), which inhibits PA activity (Figure 2B), and local generation of plasmin. Notably, the inhibition of the fibrinolysis at 23 hours was independent of the dose of the LTI-01 or the day of the treatment. Moreover, the levels PF plasminogen and active PAI-1 observed at 23 hours after the treatment were similar to those detected at baseline (Figure 3A). Thus, at all predosing intervals, plasminogen was sufficient to support plasmin generation by LTI-01 IPFT in PFs and confirmed by PF plasminogen levels (Supplemental Figure 6). The correlation of the FP, which we have identified as a companion diagnostic, with changes in opacification (Figure 3D) provides preliminary support for its application as a predictor of IPFT efficacy and justifies future testing of its ability to predict IPFT outcomes. The levels of PAI-1 antigen achieved in the PFs of patients in this study were comparable to those previously reported $(26,39,40)$. Consistent with our more recent report (18), PAI-1 activity was also markedly 
increased in these PFs. Increased levels of PF bioactiveA2M-uPA complexes at 3 hours after LTI-01 IPFT were generally identified (Figure 3C) and suggest that the mechanism of processing and bioavailability of LTI-01 IPFT used in these subjects was comparable to that of scuPA IPFT (Abbott Laboratories, glycosylated scuPA derived from S2 cells) used in our models of pleural injury and empyema $(13,18)$.

The biochemical analyses we conducted allowed us to assess how individual responses to LTI-01 IPFT impacted outcomes These analyses helped us explain the efficacy of lower dosing of LTI-01 in some patients. The favorable results in the 800,000 IU-treated group are especially encouraging. We ultimately plan to advance this dose and conduct further dose escalations as part of phase II testing. Ultimately, we hope to identify a dose of LTI-01 that is well tolerated by patients with CPE/empyema and that can be effective in almost all patients, including those with high levels of PF PAI-1 activity.

In summary, this study offers several contributions that we believe to be novel in the field. LTI-01 was safe at the doses we tested. It was bioactive in CPE/empyema PFs. Analyses of FP and processing of LTI-01 and perhaps other IPFT agents can inform understanding of treatment outcomes in individual subjects. Treatment benefits observed in some subjects support the advancement of LTI-01 to further clinical trial testing.

\section{Methods}

Involved centers. The trial included a total of 7 sites, of which 3 were in New Zealand, including Auckland City Hospital, Auckland; University of Otago, Christchurch Hospital, Christchurch; and Dunedin Hospital, Dunedin. There were 4 sites in Australia, including Westmead Hospital, Westmead, New South Wales; Cairns Hospital, Cairns, Queensland; Footscray Hospital/Western Health, Footscray, Victoria; and Sir Charles Gairdner Hospital, Nedlands, Western Australia.

Trial objectives. The primary objective was to evaluate the safety and tolerability of escalating doses of intrapleural LTI-01. Secondary objectives were to evaluate PK and PD effects of escalating doses of intrapleural LTI-01 and to assess efficacy parameters in subjects treated with escalating doses of intrapleural LTI-01. The intrapleural and systemic processing of LTI-01 was also evaluated by assessments done in $\mathrm{PF}$ and plasma obtained from each subject.

Subjects. Subjects included individuals with a clinical presentation compatible with pneumonia and parapneumonic effusion who had $\mathrm{PF}$ requiring drainage, whose pleural space failed to drain within 3 hours of tube thoracostomy, and who were at least 21 years of age. Safety precautions included exclusion of cognitively impaired and institutionalized persons, and exclusion of subjects with other disease processes that may have interfered with the interpretation of results (see Supplemental Table 2, study protocol). All subjects underwent tube thoracostomy and attempted drainage of their infected PF prior to study enrollment and were to be recruited only if their pleural space could not be drained within 3 hours after tube thoracostomy. Subjects were to receive all other customary therapy for CPE/empyema, including antibiotic therapy. The first subject was enrolled March 7, 2017, and the date the last subject was completed was March 26, 2018.

Identity of investigational product. LTI-01 was supplied as a sterile, single-use vial containing approximately $60,000 \mathrm{IU}(0.5 \mathrm{mg})$ of scuPA in a formulation of $5 \mathrm{mM}$ citrate, $25 \mathrm{mM}$ sodium chloride, and $15 \mathrm{mg} /$ $\mathrm{ml}$ mannitol, $\mathrm{pH} 4.5$. LTI-01 is a lyophilized drug product reconstituted with $1.0 \mathrm{ml}$ water for injection. The drug substance was manufactured through NHLBI SMARTT, with lyophilization and vialing completed with support provided by Lung Therapeutics Inc. The reconstituted material was administered via chest tube followed by a "chase" to bring the total injected volume (LTI-01 plus saline chase) to $30 \mathrm{ml}$.

Method of assigning subjects to dose levels. Subjects were assigned sequentially to dose level. Blinding was not done, as this was an open-label study.

Selection of doses in the study. Dosing was first based on studies in models of intrapleural injury that demonstrated therapeutic benefit and tolerance and bioavailability of LTI-01 (13, 15, 16, 41). We sought to initiate dosing at a level that was likely to be at or near a potentially therapeutic dose, since the study subjects had potentially life-threatening conditions. To balance this intention with a safe starting dose, a maximum recommended starting dose (MRSD) was calculated based on an assumption of an intrapleural no observed adverse effect level (NOAEL) of $0.25 \mathrm{mg} / \mathrm{kg}$ based on toxicology in rabbits and dogs, converting that dose to the human equivalent dose by allometrically scaling the dose based on body surface area and then adding a 10-fold safety factor to account for interspecies variation. This calculation resulted in an acceptable maximal safe starting dose of $\sim 130,000$ IU of LTI-01 for a $60-\mathrm{kg}$ subject. To be conservative, we opted to initiate at a lower dose to assess potential effects of vehicle and impurities. 
Trial methodology. This was a prospective, open-label, dose escalation safety trial. Eligible subjects began treatment with intrapleural LTI-01 within approximately 24 hours of initial consent and enrollment. Treatment was administered as a bolus dose through the chest tube into the pleural space and allowed to stay in the space for 3 hours. Treatment was given once per day for up to 3 days. A dose escalation design was used, with up to 5 dose escalation cohorts as shown in Supplemental Table 2, study protocol. Following the review of safety data from cohort A (50,000 IU LTI-01) subjects by a safety review committee (SRC), the dose level was escalated (doubled) in each consecutive group of 3 subjects with dosing for up to 3 days at all dose levels. A total of 21 patients could have been enrolled.

The study protocol permitted completion of dosing after $<3$ daily doses with complete resolution of the pleural process. Bleeding events were defined as an acute bleeding event with $\mathrm{a} \geq 2 \mathrm{~g} / \mathrm{dl}$ drop in blood hemoglobin; or development of melena, hemothorax, or PF/blood hematocrit $>50 \%$.

All subjects at all dose levels were observed for 4 days after last intrapleural LTI-01 treatment (or until hospital discharge if sooner). Clinical stability was to be documented before treatment of the next subject. Blood and PF assessments included complete blood counts (CBCs), coagulation and metabolic profiles, and fibrinogen and CRP levels done before and at 3 and 23 hours after dosing. Chest X-ray and CT scans and other safety assessments were also performed before and at about 4 days after administration of LTI-01.

Blood and PF cultures were obtained, as well as other diagnostic tests deemed appropriate, including atypical pneumonia serology and pneumococcal antigen at screening. Procedures included chest X-ray and CT imaging and measurement of chest tube drainage, chest tube flushes, and drain suction. Use of alternative forms of IPFT or referral for surgery was to occur as needed after completion of administration of LTI-01 unless circumstances necessitated earlier institution.

A displaced thoracostomy tube was only to be replaced if clinically indicated for PF drainage and not solely for trial drug administration. If the chest tube displacement occurred over a period of less than 48 hours, the trial treatment course could be restarted and completed, after chest tube replacement, if clinically indicated to complete the planned LTI-01 IPFT. Study drug administration was to be delayed if the prothrombin time/partial thromboplastin time (PT/PTT) was $>1.7 \times$ control or PT $>15$ seconds, or fibrinogen was $<150 \mathrm{mg} / \mathrm{dl}$, at the last available time point prior to dose administration (typically within 3 hours prior to dose administration). To militate against these changes, prophylaxis for DVT was delivered as far removed as possible (e.g., 9-12 hours) from IPFT. Continued dosing was only allowed if the PT/PTT was $\leq 1.7$ and $\mathrm{PT}<15$ seconds and fibrinogen was $>150 \mathrm{mg}$ / d1. In otherwise stable subjects, transfusion for downward drift of blood hemoglobin that may have reflected marrow suppression as a result of acute illness, hemodilution, or IPFT was not considered as a stopping criterion.

Safety assessments including adverse events. SI served on the Safety Review Committee with JG and GS, and John Wheatley, Westmead Hospital, and John Kolbe, Auckland City Hospital. Subjects were monitored for all TEAEs starting prior to the first study dose (day 1 at the time of initiation of administration of dose 1) until 23 hours after last dose of LTI-01. At each contact with the subject, information regarding adverse events was elicited by appropriate questioning and examinations. Grade III and grade IV adverse events were collected from the time of first study dose through to day 7 or hospital discharge, whichever came first. SAEs were collected from the time of first study dose through day 28, hospital discharge, or death, whichever occurred first. Adverse events overall are listed in Supplemental Table 3.

Efficacy endpoints of the study. Efficacy endpoints were determined based on monitoring of vital signs, referrals for surgery, mortality during hospitalization, and radiographic improvement based on comparison of pretreatment chest X-ray or CT scan with those taken $23 \pm 3$ hours after the last received daily dose of LTI-01 IPFT, as previously reported (5). The first radiographic study was an upright 2-view chest X-ray. Pleural effusion was confirmed radiographically. If pleural loculation was suggested on the plain chest X-ray, it was confirmed using CT scanning of the chest or chest ultrasonography. CT scanning (high-resolution if available) was done unless precluded by the clinical status of the subject or unavailability of the test. At $23 \pm$ 3 hours after the third (or final) dose of LTI-01, PF-related density was compared by follow-up chest X-ray, with relative change in pleural opacification versus baseline imaging, as previously described (5). Days of hospitalization for this episode of CPE/empyema, total PF drainage from an initial dose of LTI-01 IPFT until 23 hours after the last LTI-01 IPFT dose versus baseline, stability or decline of white and neutrophil cell counts in blood and CRP levels in plasma were also assessed. The volume of drainage from the chest tube was documented daily for up to 23 hours after last dose and totaled for the full course (up to 3 days) of treatment. 
Central laboratory analyses. These studies were done at UTHSCT and included D-dimer and PA as well as fibrinolytic activity levels in plasma and PFs. Levels of PAI-1 antigen and activity levels were also assessed (18), as were levels of bioactive A2M-uPA complexes (13). FP measurement of fibrinolytic activity after supplementation of baseline PFs with 2-chain UPA was determined in samples of PF collected prior to the IPFT as previously described $(21,22)$. A total of $n=8$ PFs were available for these analyses.

$P K$ and $P D$ analyses. These included total UPA antigen PK and PD in plasma and PFs. A uPA ELISA/ Quantikine ELISA (urokinase, R\&D Systems, DUPA00, lot 171962) was used to perform these studies. The assay measures uPA-related antigen and detects single-chain uPA, 2-chain uPA, uPA-PAI-1 complexes, and uPA bound to its receptor (UPA-uPAR complexes). Samples of PF and plasma were collected at the intervals stipulated, using methods appropriate for these assessments (Supplemental Table 4).

Prior and concomitant therapy. Subjects were expected to receive customary care, including radiologic chest tube placement and initiation of antibiotics prior to enrollment. Radiologic tube placement, while not required, was considered justified, as large bore tubes had not been proven to be more effective and were associated with greater morbidity, and radiologically placed tubes had the advantage of being located within the PF collection. Subjects could receive all other customary therapy for CPE/empyema. Antibiotic regimens used were at the discretion of the managing physician.

Subject follow-up. At 3 and 12 months after treatment, attempts were made to document surgical referral, further hospitalization for CPE/empyema, and mortality in treated subjects through the outpatient clinic.

Statistics. All statistical analyses were exploratory in nature, with descriptive statistics (i.e., mean, SD, median, maximum, and minimum) provided for continuous variables. Categorical variables are summarized by frequency distributions (number and percentage of subjects). The ITT population comprised all subjects who enrolled in the study and who provided any post-screening data. Pairwise comparisons depicted in Figures 2 and 3 were done using Wilcoxon's matched-pairs signed-rank test. Because we assumed that the $\mathrm{PF}$ and plasma environments differed each day as a result of different durations of antibiotics and other treatments, pairwise comparison of separate samples was done. Thus, no correction for multiple comparisons was warranted. Correlations were assessed using Spearman's rank correlation analysis (depicted in Figure 3). $P$ values less than 0.05 were considered statistically significant.

Study approval and registration. Written informed consent was received from all participants prior to inclusion in the study. Ethics approvals were obtained from all required organizations, including (i) the Western Sydney Local Health District Human Research Ethics Committee, Westmead Hospital; (ii) Sir Charles Gairdner and Osborne Park Health Care Group Human Research Ethics Committee, Nedlands; and (iii) Northern B Health and Disability Ethics Committee, Ministry of Health, Wellington, New Zealand. This trial complied with the ethical principles of Good Clinical Practice (GCP) and International Council for Harmonisation of Technical Requirements for Pharmaceuticals for Human Use (ICH) guidelines, and with the Declaration of Helsinki. Subjects had the right to refuse participation without explanation and could withdraw at any time without affecting their follow-up care. In addition, clinicians managing the subject could choose alternative treatment at any time in the best interest of the given subject. Medical confidentiality was preserved, and personal identifying information was not revealed.

\section{Author contributions}

LB, BB, GS, AMS, and YCGL served as site principal investigators and recruited/managed patients enrolled in this study. SI, YGCL, RWL, and NR helped to design and review the protocol. JG and SI served of the Safety Review Committee along with representatives of the Australian and New Zealand sites. JG served as medical monitor. SS and SI reviewed and organized the data, including that from the clinical study report (CSR). KPS, HN, and GF performed statistical analyses. AAK and GF coordinated and with KS performed the sample biochemical analyses. AAK and GF analyzed, plotted, and interpreted these data. TO and WB independently performed and with SS prepared the imaging analyses. SI, SS, AAK, GF, and NR prepared the manuscript, which was reviewed by all authors.

\section{Acknowledgments}

NIH SMARTT HHSN268201100014C (SI) provided funding of the manufacturing of the drug substance, toxicology studies, and regulatory support; NIH UO-1 HL121841-01A1 (SI) provided initial funding in preparation for the clinical trial. 
Address correspondence to: Steven Idell, The University of Texas Health Science Center at Tyler, 11937 US HWY 271, Tyler, Texas 75708, USA. Phone: 903.877.7556; Email: steven.idell@uthct.edu.

1. Grijalva CG, Zhu Y, Nuorti JP, Griffin MR. Emergence of parapneumonic empyema in the USA. Thorax. 2011;66 (8):663-668.

2. Farjah F, Symons RG, Krishnadasan B, Wood DE, Flum DR. Management of pleural space infections: a population-based analysis. J Thorac Cardiovasc Surg. 2007;133 (2):346-351.

3. Desrumaux A, et al. Épidémiologie et caractéristiques cliniques des complications suppuratives des pneumonies de l'enfant [Epidemiology and clinical characteristics of childhood parapneumonic empyemas]. Arch Pediatr. 2007;14(11):1298-1303.

4. Maskell NA, et al. U.K. Controlled trial of intrapleural streptokinase for pleural infection. N Engl J Med. 2005;352 (9):865-874

5. Rahman NM, et al. Intrapleural use of tissue plasminogen activator and DNase in pleural infection. $N$ Engl J Med. 2011;365(6):518-526

6. Corcoran JP, Hallifax R, Rahman NM. New therapeutic approaches to pleural infection. Curr Opin Infect Dis. 2013;26 (2):196-202.

7. Tillett WS, Sherry S. The effect in patients of streptococcal fibrinolysin (streptokinase) and streptococcal desoxyribonuclease on fibrinous, purulent, and sanguinous pleural exudations. J Clin Invest. 1949;28 (1):173-190.

8. Janda S, Swiston J. Intrapleural fibrinolytic therapy for treatment of adult parapneumonic effusions and empyemas: a systematic review and meta-analysis. Chest. 2012;142 (2):401-411.

9. Cameron R, Davies HR. Intra-pleural fibrinolytic therapy versus conservative management in the treatment of adult parapneumonic effusions and empyema. Cochrane Database Syst Rev. 2008;(2):CD002312.

10. Redden MD, Chin TY, van Driel ML. Surgical versus non-surgical management for pleural empyema. Cochrane Database Syst Rev. 2017;3:CD010651.

11. Tucker T, Idell S. Plasminogen-plasmin system in the pathogenesis and treatment of lung and pleural injury. Semin Thromb Hemost. 2013;39 (4):373-381.

12. Piccolo F, Popowicz N, Wong D, Lee YC. Intrapleural tissue plasminogen activator and deoxyribonuclease therapy for pleural infection. J Thorac Dis. 2015;7(6):999-1008.

13. Komissarov AA, Mazar AP, Koenig K, Kurdowska AK, Idell S. Regulation of intrapleural fibrinolysis by urokinase-alpha-macroglobulin complexes in tetracycline-induced pleural injury in rabbits. Am J Physiol Lung Cell Mol Physiol. 2009;297 (4):L568-L577.

14. Idell S, et al. Single-chain urokinase in empyema induced by Pasturella multocida. Exp Lung Res. 2009;35 (8):665-681.

15. Idell S, Allen T, Chen S, Koenig K, Mazar A, Azghani A. Intrapleural activation, processing, efficacy, and duration of protection of single-chain urokinase in evolving tetracycline-induced pleural injury in rabbits. Am J Physiol Lung Cell Mol Physiol. 2007;292 (1):L25-L32.

16. Idell S, et al. Single-chain urokinase alone or complexed to its receptor in tetracycline-induced pleuritis in rabbits. Am J Respir Crit Care Med. 2002;166(7):920-926.

17. Karandashova S, et al. Intrapleural adenoviral delivery of human plasminogen activator inhibitor-1 exacerbates tetracycline-induced pleural injury in rabbits. Am J Respir Cell Mol Biol. 2013;48 (1):44-52.

18. Komissarov AA, et al. Dose dependency of outcomes of intrapleural fibrinolytic therapy in new rabbit empyema models. $A m J$ Physiol Lung Cell Mol Physiol. 2016;311 (2):L389-L399.

19. Idell S, Rahman NM. Intrapleural fibrinolytic therapy for empyema and pleural loculation: knowns and unknowns. Ann Am Thorac Soc. 2018;15 (5):515-517.

20. Komissarov AA, et al. Fibrin turnover and pleural organization: bench to bedside. Am J Physiol Lung Cell Mol Physiol. 2018;314 (5):L757-L768

21. Florova G, et al. Targeting of plasminogen activator inhibitor 1 improves fibrinolytic therapy for tetracycline-induced pleural injury in rabbits. Am J Respir Cell Mol Biol. 2015;52 (4):429-437.

22. Idell S, et al. Precision-guided, personalized intrapleural fibrinolytic therapy for empyema and complicated parapneumonic pleural effusions: the case for the fibrinolytic potential. Clin Pulm Med. 2017;24 (4):163-169.

23. Tillett WS, Sherry S, Read CT. The use of streptokinase-streptodornase in the treatment of postneumonic empyema. J Thorac Surg. 1951;21 (3):275-297.

24. Lee YC, Idell S, Stathopoulos GT. Translational research in pleural infection and beyond. Chest. 2016;150 (6):1361-1370.

25. Popowicz N, Idell S, Lee YCG. Pathogenesis of pleural infection: a complex warfare. Respirology. 2018;23 (1):8-9.

26. Philip-Joët F, et al. Fibrinolytic and inflammatory processes in pleural effusions. Eur Respir J. 1995;8 (8):1352-1356.

27. Chiu CY, Wong KS, Huang JL, Tasi MH, Lin TY, Hsieh SY. Proinflammatory cytokines, fibrinolytic system enzymes, and biochemical indices in children with infectious para-pneumonic effusions. Pediatr Infect Dis J. 2008;27 (8):699-703.

28. Alemán C, et al. Intrapleural fibrinolysis with urokinase versus alteplase in complicated parapneumonic pleural effusions and empyemas: a prospective randomized study. Lung. 2015;193 (6):993-1000.

29. Majid A, et al. Concurrent intrapleural instillation of tissue plasminogen activator and DNase for pleural infection. A single-center experience. Ann Am Thorac Soc. 2016;13 (9):1512-1518

30. Popowicz N, et al. Dose de-escalation of intrapleural tissue plasminogen activator therapy for pleural infection. The Alteplase Dose Assessment for Pleural Infection Therapy Project. Ann Am Thorac Soc. 2017;14 (6):929-936.

31. Florova G, et al. Targeting plasminogen activator inhibitor-1 in tetracycline-induced pleural injury in rabbits. Am J Physiol Lung Cell Mol Physiol. 2018;314(1):L54-L68.

32. Komissarov AA, et al. The time course of resolution of adhesions during fibrinolytic therapy in tetracycline-induced pleural injury in rabbits. Am J Physiol Lung Cell Mol Physiol. 2015;309(6):L562-L572.

33. Idell S. Update on the use of fibrinolysins in pleural disease. Clinical Pulmonary Medicine. 2005;12(3):184-190.

34. Gervais DA, Levis DA, Hahn PF, Uppot RN, Arellano RS, Mueller PR. Adjunctive intrapleural tissue plasminogen activator administered via chest tubes placed with imaging guidance: effectiveness and risk for hemorrhage. Radiology. 2008;246 (3):956-963

35. Anevlavis S, et al. Intrapleural r-tPA in association with low-molecular heparin may cause massive hemothorax resulting in hypovolemia. Respiration. 2011;81 (6):513-516. 
36. National Clinical Guideline Centre (UK). Blood Transfusion. NICE Guideline, no. 24. London, United Kingdom: National Institute for Health and Care Excellence; 2015. https://www.ncbi.nlm.nih.gov/pubmed/26632625. Accessed April 17, 2019.

37. Guth MC, Kaufner L, Kleber C, von Heymann C. Behandlung der traumainduzierten Koagulopathie - Was ist die Evidenz? [Therapy of trauma-induced coagulopathy - what is the evidence?]. Anasthesiol Intensivmed Notfallmed Schmerzther. 2012;47(9):528-539.

38. Schlimp CJ, Schöchl H. The role of fibrinogen in trauma-induced coagulopathy. Hamostaseologie. 2014;34(1):29-39.

39. Idell S, Girard W, Koenig KB, McLarty J, Fair DS. Abnormalities of pathways of fibrin turnover in the human pleural space. Am Rev Respir Dis. 1991;144 (1):187-194.

40. Chung CL, Chen CH, Sheu JR, Chen YC, Chang SC. Proinflammatory cytokines, transforming growth factor-beta1, and fibrinolytic enzymes in loculated and free-flowing pleural exudates. Chest. 2005;128 (2):690-697.

41. Idell S, et al. Intrapleural low-molecular-weight urokinase or tissue plasminogen activator versus single-chain urokinase in tetracycline-induced pleural loculation in rabbits. Exp Lung Res. 2007;33(8-9):419-440. 